\title{
Glucagon-Like Peptide-1 Receptor Agonist Inhibits Asymmetric Dimethylarginine Generation in the Kidney of Streptozotocin-Induced Diabetic Rats by Blocking Advanced Glycation End Product-Induced Protein Arginine Methyltranferase-1 Expression
}

\author{
Ayako Ojima, ${ }^{*}$ Yuji Ishibashi, ${ }^{\star}$ Takanori Matsui, ${ }^{*}$ Sayaka Maeda, ${ }^{*}$ Yuri Nishino, ${ }^{*}$ Masayoshi Takeuchi, ${ }^{\dagger}$ Kei Fukami, ${ }^{\ddagger}$ and \\ Sho-ichi Yamagishi*
}

From the Departments of Pathophysiology and Therapeutics of Diabetic Vascular Complications* and Medicine, ${ }^{\ddagger}$ Kurume University School of Medicine, Kurume; and the Department of Advanced Medicine, ${ }^{\dagger}$ Medical Research Institute, Kanazawa Medical University, Kanazawa, Japan

\author{
Accepted for publication \\ September 25, 2012. \\ Address correspondence to \\ Sho-ichi Yamagishi, M.D., \\ Department of Pathophysiology \\ and Therapeutics of Diabetic \\ Vascular Complications, \\ Kurume University School of \\ Medicine, 67 Asahi-machi, \\ Kurume 830-0011, Japan. \\ E-mail: shoichi@med.kurume-u. \\ ac.jp.
}

\begin{abstract}
Advanced glycation end products (AGEs) and their receptor (RAGE) play a role in diabetic nephropathy. Asymmetric dimethylarginine (ADMA), an endogenous inhibitor of nitric oxide synthase, contributes to diabetic nephropathy. We have found that glucagon-like peptide-1 (GLP-1) inhibits the AGE-induced inflammatory reactions in endothelial cells. However, effects of GLP-1 on the AGE-RAGE-ADMA axis are unknown. This study examined the effects of GLP-1 on reactive oxygen species (ROS) generation, gene expression of protein arginine methyltransfetase-1 (PRMT-1), an enzyme that mainly generates ADMA, and ADMA levels in human proximal tubular cells. Streptozotocin-induced diabetic rats received continuous i.p. infusion of $0.3 \mu \mathrm{g}$ of vehicle or $1.5 \mu \mathrm{g}$ of the GLP-1 analog exendin- 4 per kilogram of body weight for 2 weeks. We further investigated whether and how exendin- 4 treatment reduced ADMA levels and renal damage in streptozotocin-induced diabetic rats. GLP-1 inhibited the AGE-induced RAGE and PRMT-1 gene expression, ROS, and ADMA generation in tubular cells, which were blocked by small-interfering RNAs raised against GLP-1 receptor. Exendin-4 treatment decreased gene expression of Rage, Prmt-1, Icam-1, and Mcp-1 and ADMA level; reduced urinary excretions of 8-hydroxy-2'-deoxyguanosine and albumin; and improved histopathologic changes of the kidney in diabetic rats. Our present study suggests that GLP-1 receptor agonist may inhibit the AGE-RAGE-mediated ADMA generation by suppressing PRMT-1 expression via inhibition of ROS generation, thereby protecting against the development and progression of diabetic nephropathy. (Am J Pathol 2013, 182: 132-141; http:// dx.doi.org/10.1016/j.ajpath.2012.09.016)
\end{abstract}

The pathologic role of the nonenzymatic modification of proteins by reducing sugars, a process that is known as glycation (also called the Maillard reaction), has become increasingly evident in various types of diseases. ${ }^{1-3}$ It is now well established that early glycation products undergo progressive modification over time in vivo to the formation of irreversible cross-links, after which these molecules are termed "advanced glycation end products" (AGEs). ${ }^{1-3}$ The formation and accumulation of AGEs in various tissues have been reported to progress at an accelerated rate under hyperglycemic conditions. ${ }^{1-3}$ There is a growing body of evidence that AGE and receptor for AGE (RAGE) interaction stimulates oxidative stress generation and subsequently evokes inflammatory reactions, thereby causing progressive alteration in renal architecture and loss of renal function in

Supported in part by Grants of Venture Research and Development Centers from the Ministry of Education, Culture, Sports, Science and Technology, Japan (S.Y.). 
diabetes. ${ }^{4-8}$ RAGE-overexpressing diabetic mice have been found to have progressive glomerulosclerosis with renal dysfunction, compared with diabetic littermates lacking the $R A G E$ transgene. ${ }^{4}$ Diabetic homozygous $R A G E$ null mice failed to develop significantly increased mesangial matrix expansion or thickening of the glomerular basement membrane. ${ }^{5}$ Furthermore, deletion of RAGE is also reported to prevent diabetic nephropathy in the OVE26 type 1 mouse, a model of progressive glomerulosclerosis, and decline of renal function. ${ }^{6}$

Glucagon-like peptide-1 (GLP-1) is one of the incretins, a gut hormone secreted from $\mathrm{L}$ cells in the intestine in response to food intake. ${ }^{9}$ GLP-1 augments glucose-induced insulin release from pancreatic $\beta$-cells, suppresses glucagon secretion, and slows gastric emptying. ${ }^{9}$ Therefore, GLP-1 has been proposed as a potential therapeutic target for the treatment of patients with type 2 diabetes. The biological actions of GLP-1 on pancreatic cells are mainly mediated by the high-affinity GLP-1 receptor (GLP-1R). ${ }^{10}$ GLP-1R exists in extrapancreatic tissues, including brain, peripheral nervous system, kidney, heart, and vasculature. $^{11,12}$ These observations suggest that GLP-1 could exert pleiotropic actions, that is, glucose-lowering independent beneficial effects on vascular complications in diabetes via GLP-1R-mediated activation of cAMP pathway.

Nitric oxide (NO) is a multifunctional molecule critical to a number of physiologic and pathologic processes in humans. ${ }^{13,14}$ NO not only inhibits inflammatoryproliferative reactions in vascular wall cells but also exerts antithrombogenic and endothelial cell protective properties in vivo. ${ }^{13,14}$ Therefore, impaired production and/or bioavailability of NO are considered to play a role in vascular complications in diabetes, such as diabetic nephropathy and cardiovascular disease. ${ }^{15,16}$ Indeed, circulating level of asymmetric dimethylarginine (ADMA), an endogenous NO synthase inhibitor, is increased in early diabetic nephropathy in type 1 diabetes and associated with future cardiovascular events in these individuals. ${ }^{17}$ Furthermore, we have previously found that serum levels of AGEs are positively associated with soluble form of RAGE and ADMA in patients with chronic kidney disease, thus suggesting the active involvement of the AGE-RAGE system in the elevated levels of ADMA. ${ }^{18}$ However, it remains unknown how the AGE-RAGE interaction increases ADMA levels in diabetic kidney and whether GLP-1 could reduce the ADMA levels by suppressing the AGE-RAGE axis. To address these issues, we examined the effects of GLP-1 on RAGE mRNA levels, reactive oxygen species (ROS) generation, gene expression of protein arginine methyltransfetase-1 (PRMT-1), and dimethylarginine dimethylaminohydrolase-1 (DDAH-1), responsible enzymes that mainly generate and metabolize ADMA,${ }^{15}$ and ADMA levels in human proximal tubular epithelial cells. Then we investigated whether continuous intraperitoneal infusion of the GLP-1 analog exendin- 4 could decrease ADMA levels in the kidney of streptozotocin-induced diabetic rats and inhibit renal damage in these animals.

\section{Materials and Methods}

\section{Materials}

Bovine serum albumin (BSA) (essentially fatty acid free and essentially globulin-free, lyophilized powder), GLP-1 (7 to 36) amide, 8-bromo-cAMP (8-Br-cAMP; an analog of cAMP), $N$-acetylcysteine (NAC), monoclonal antibody (Ab) raised against $\alpha$-tubulin, and exendin-4 (GLP-1 analog) were purchased from Sigma Chemical Co. (St. Louis, MO). D-glyceraldehyde was purchased from Nakalai Tesque (Kyoto, Japan). Abs directed against human GLP-1R and ADMA were purchased from Santa Cruz Biotechnology Inc. (Delaware, CA) and Active Motif Inc. (Carlsbad, CA), respectively.

\section{Preparation of AGE-BSA}

AGE-BSA was prepared as described previously. ${ }^{19,20}$ In brief, BSA $(25 \mathrm{mg} / \mathrm{mL})$ was incubated under sterile conditions with $0.1 \mathrm{~mol} / \mathrm{L}$ glyceraldehyde in $0.2 \mathrm{~mol} / \mathrm{L} \mathrm{NaPO}_{4}$ buffer ( $\mathrm{pH}$ 7.4) for 7 days. Then unincorporated sugars were removed by PD-10 column chromatography and dialysis against PBS. Control nonglycated BSA was incubated in the same conditions except for the absence of reducing sugars. Preparations were tested for endotoxin using Endospecy ES20S system (Seikagaku Co., Tokyo, Japan); no endotoxin was detectable. The extent of chemical modification was determined as described with 2,4,6-trinitrobenzenesulfonic acid as a difference in lysine residues of modified and unmodified protein preparations. ${ }^{13,14}$ The extent of lysine modification of modified BSA preparations was $65 \%$ for AGE-BSA. ${ }^{21}$

\section{Preparation of AGE and RAGE Abs}

Polyclonal Ab raised against AGE-BSA was prepared as previously described. ${ }^{19}$ Ab directed against human RAGE (RAGE-Ab) for neutralizing assays, which recognized the amino acid residues 167 to 180 of human RAGE protein, was prepared as described previously. ${ }^{22}$

\section{Cells}

Proximal tubular epithelial cells from human kidney were maintained in basal medium supplemented with $5 \%$ fetal bovine serum, $0.5 \mu \mathrm{g} / \mathrm{mL}$ of hydrocortisone, $10 \mathrm{ng} / \mathrm{mL}$ of human epidermal growth factor, $0.5 \mu \mathrm{g} / \mathrm{mL}$ of epinephrine, $6.5 \mathrm{ng} / \mathrm{mL}$ of triiodo-L-thyronine, $10 \mu \mathrm{g} / \mathrm{mL}$ of transferrin, $5 \mu \mathrm{g} / \mathrm{mL}$ of insulin, and GA-1000 according to the supplier instructions (Clonetics Corp., San Diego, CA) ${ }^{23}$ Cells at three to five passages were used for the experiments. AGE and/or GLP-1 treatments were performed in a serum-free 
basal medium containing $10 \mu \mathrm{g} / \mathrm{mL}$ of transferrin and GA-1000.

\section{Construction and Transfection of siRNAs}

Sense and antisense human GLP-1R small-interfering RNAs (siRNAs) (GLP-1R siRNA) used in this experiment $\left(5^{\prime}\right.$ UCAUCAAGCUGUUUACAGATT- ${ }^{\prime}$ and $5^{\prime}$-UCUGUAAA CAGCUUGAUGAAG- $3^{\prime}$, respectively) were synthesized by Applied Biosystems (Foster, CA). Control nonsilencing siRNAs (control siRNA) were also obtained from Applied Biosystems (Silencer Negative Control 1 siRNA). Then the siRNA duplexes were transfected to tubular cells using Lipofectamine2000 (Invitrogen, Carlsbad, CA) as described previously. ${ }^{24}$ After 2 days of transfection, GLP-1R protein levels were analyzed.

\section{Real-Time RT-PCR}

Transfected or nontransfected tubular cells were treated with $100 \mu \mathrm{g} / \mathrm{mL}$, AGE-BSA, or nonglycated BSA in the presence or absence of $0.3 \mathrm{nmol} / \mathrm{L}$ GLP-1, $5 \mu \mathrm{mol} / \mathrm{L}$ 8-Br-cAMP, $5 \mu \mathrm{g} / \mathrm{mL}$ of RAGE-Ab, or $1 \mathrm{mmol} / \mathrm{L}$ NAC for 4 hours. Then total RNA was extracted with the RNAqueous-4PCR kit (Ambion Inc., Austin, TX) according to the manufacturer instructions. Quantitative real-time RT-PCR (RT-qPCR) was performed using Assay-on-Demand and TaqMan 5 fluorogenic nuclease chemistry (Applied Biosystems) according to the supplier recommendation. The identification numbers of primers for human RAGE, PRMT-1, and $\beta$-actin (ACTB) genes were Hs00153957_m1, Hs01587651_g1, and Hs99999903_m1, respectively.

\section{Western Blotting Analysis}

Proteins were extracted from transfected or nontransfected tubular cells with lysis buffer and then separated by SDSPAGE and transferred to nitrocellulose membranes as described previously. ${ }^{25}$ Membranes were probed with Abs against GLP-1R, ADMA, or $\alpha$-tubulin, and then immune complexes were visualized with an enhanced chemiluminescence detection system (Amersham Bioscience, Buckinghamshire, United Kingdom).

\section{Measurement of Superoxide Generation with DHE Staining}

Transfected or nontransfected tubular cells were treated with $100 \mu \mathrm{g} / \mathrm{mL}$ of AGE-BSA or nonglycated BSA in the presence or absence of $0.3 \mathrm{nmol} / \mathrm{L} \mathrm{GLP}-1$ for 4 hours, and then the cells were incubated with phenol red free Dulbecco's modified Eagle medium containing $3 \mu \mathrm{mol} / \mathrm{L}$ dihydroethidium (DHE; Molecular Probes Inc., Eugene, OR). After 15 minutes, the cells were imaged under a laser scanning confocal microscope. Intensity of DHE staining in five different fields of each sample was analyzed by microcomputer-assisted ImageJ software version 1.46 (NIH, Bethesda, MD).
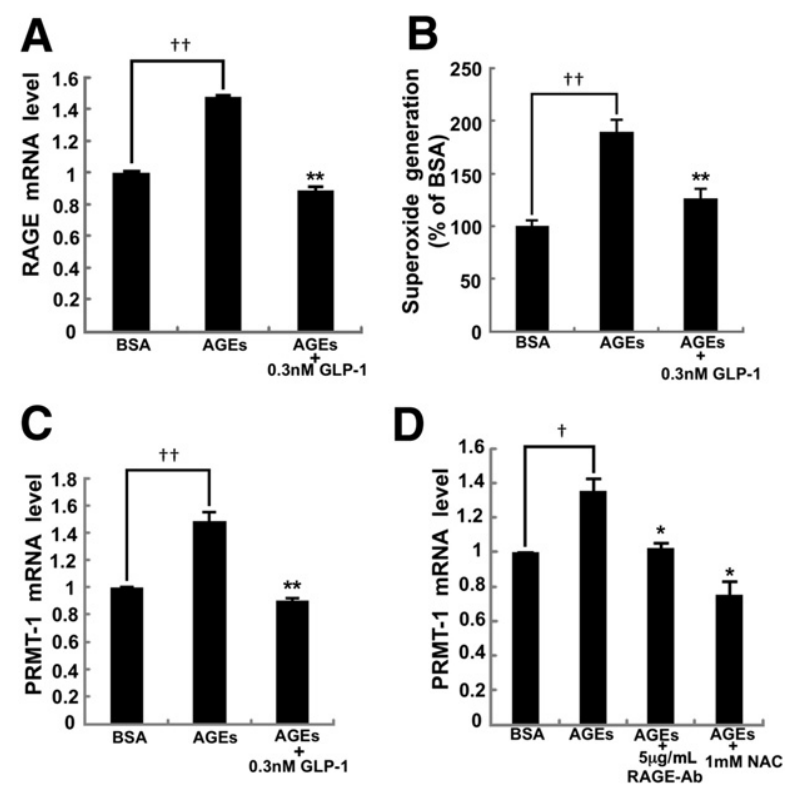

Figure 1 Effects of GLP-1 on RAGE gene expression (A), superoxide generation (B), and PRMT-1 mRNA level in tubular cells. Cells were treated with $100 \mu \mathrm{g} / \mathrm{mL}$ of AGE-BSA or nonglycated BSA in the presence or absence of $0.3 \mathrm{nmol} / \mathrm{L} \mathrm{GLP}-1,5 \mu \mathrm{g} / \mathrm{mL}$ of RAGE-Ab, or $1 \mathrm{mmol} / \mathrm{L} \mathrm{NAC}$ for 4 hours. A, C, and $\mathbf{D}$ : Total RNAs were transcribed and amplified by real-time PCR. Data were normalized by the intensity of $\beta$-actin mRNA-derived signals and then related to the value obtained with nonglycated BSA ( $n=3$ to 6 per group). B: Cells were incubated with DHE. Panel shows quantitative data of superoxide generation evaluated by fluorescent intensity ( $n=10$ per group). ${ }^{*} P<$ $0.05, * * P<0.01$ compared with the value with AGEs alone, respectively; ${ }^{\dagger} P<0.05$ and ${ }^{\dagger} P<0.01$ compared with the value with BSA, respectively.

\section{Immunostaining of ADMA}

Transfected or nontransfected tubular cells were treated with $100 \mu \mathrm{g} / \mathrm{mL}$ of AGE-BSA or nonglycated BSA in the presence or absence of $0.3 \mathrm{nmol} / \mathrm{L}$ GLP-1 for 4 hours; the cells were then fixed with $4 \%$ paraformaldehyde and washed with PBS. The cells were stained with rabbit $\mathrm{Ab}$ raised against human ADMA. ADMA was visualized with Alexa Fluor 488 goat anti-rabbit IgG (Molecular Probes). Intensity of ADMA staining in five different fields of each sample was analyzed by microcomputer-assisted ImageJ.

\section{Animal Experiments}

Six-week-old male Wistar rats received a single $60-\mathrm{mg} / \mathrm{kg}$ i.p. injection of streptozotocin (Sigma) in $10 \mathrm{mmol} / \mathrm{L}$ citrate buffer $(\mathrm{pH} 4.5)$. Nondiabetic control rats received citrate buffer alone. Animals with blood glucose levels $>250 \mathrm{mg} / \mathrm{dL}$ 48 hours later were considered diabetic. Streptozotocininduced diabetic rats received continuous i.p. infusion of vehicle, $0.3 \mu \mathrm{g} / \mathrm{kg} / \mathrm{h}$ of exendin- 4 , or $1.5 \mu \mathrm{g} / \mathrm{kg} / \mathrm{h}$ of exendin-4 using an osmotic mini pump (ALZET microosmotic pump model 1002; Durect Corporation, Cupertino, CA). After 2 weeks, animals were housed in metabolic cages to collect urine for measurement of urinary excretion levels of albumin and 8-hydroxy-2'-deoxyguanosine 
A

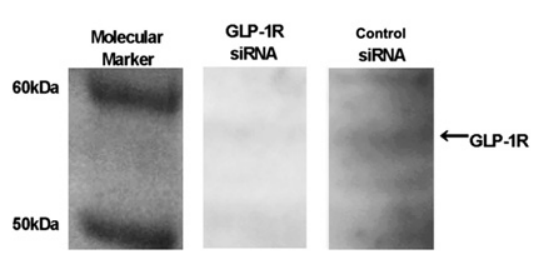

D

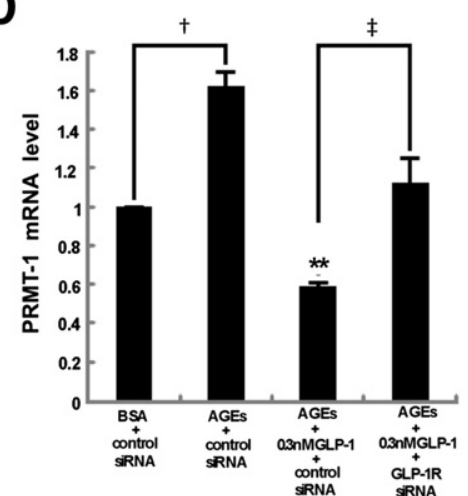

B

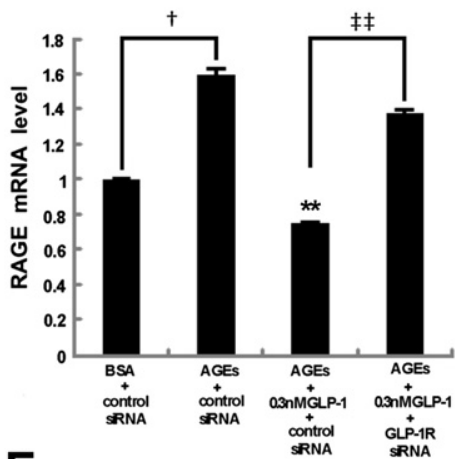

E

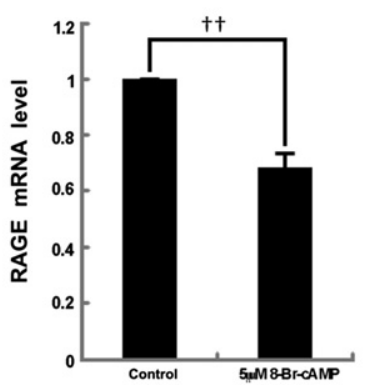

C

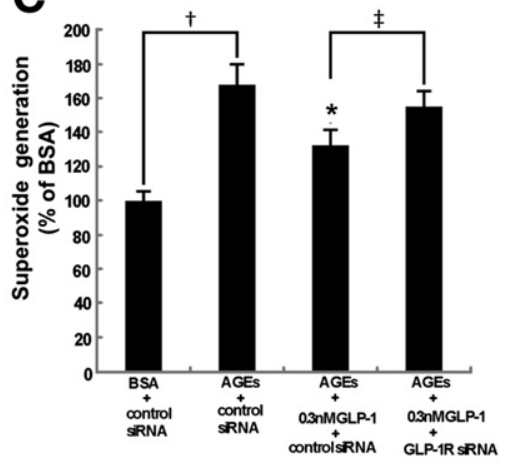

$\mathbf{F}$

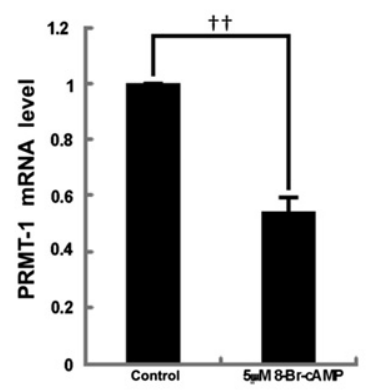

Figure 2 Effects of GLP-1R siRNA or 8-Br-cAMP on GLP-1R expression (A), RAGE gene expression (B and E), superoxide generation (C), and PRMT-1 mRNA level (D and $\mathbf{F}$ ) in tubular cells. Transfected or nontransfected cells were treated with or without $100 \mu \mathrm{g} / \mathrm{mL}$ of AGE-BSA or nonglycated BSA in the presence or absence of $0.3 \mathrm{nmol} / \mathrm{L} \mathrm{GLP}-1$ or $5 \mu \mathrm{mol} / \mathrm{L} 8$-Br-cAMP for 4 hours. Then GLP-1R protein level, RAGE, and PRMT-1 gene expression and superoxide generation were measured. A: Representative bands of Western blots. Three independent experiments obtained the same results. $[n=10$ per group (C) and $n=3$ to 6 per group (B, D-F)]. ${ }^{*} P<0.05,{ }^{* *} P<0.01$ compared with the value with AGEs and control siRNA, respectively; ${ }^{\dagger} P<0.01$ compared with the value with BSA plus control siRNA; ${ }^{\dagger \dagger} P<$ 0.01 compared with the value with control; ${ }^{\ddagger} P<0.05,{ }^{\ddagger \ddagger} P<0.01$ compared with the value with AGEs plus $0.3 \mathrm{nmol} / \mathrm{L}$ GLP-1 plus control siRNA, respectively.

(8-OHdG), and then body weight, heart rate, and blood pressure were measured. Albuminuria and urinary excretion level of 8-OHdG were determined with commercially available enzyme-linked immunosorbent assay (ELISA) kits (Exocell, Philadelphia, PA, and NIKKEN SEIL Co. Ltd., Shizuoka, Japan). Then the rats were euthanized and the kidneys were excised for real-time RT-PCR, Western blotting, and morphologic analyses. Blood chemical analyses were performed with standard enzymatic methods as described previously. ${ }^{26}$

Identifications of TaqMan primers for rat Rage, intercellular adhesion molecule 1 (Icam-1), monocyte chemoattractant protein 1 (Mcp-1), $\beta$-actin, glyceraldehyde3-phosphate dehydrogenase (GAPDH), and 18S gene were Rn00584249_m1, Rn00564227_m1, Rn00580555_m1, Rn00667869_m1, Rn99999916_s1, and Hs99999901_s1, respectively. RT-qPCR analysis for rat Prmt-1 was performed using SYBR Green reagent (Applied Biosystems) according to the manufacturer recommendation. The forward and reverse primers for rat Prmt-1 were 5'-AACTGAAGCTCGCACTCTCG- $3^{\prime}$ and $5^{\prime}$-TCAGCACAGATCTCCTTGGC- $3^{\prime}$. ADMA and AGE levels in the kidney were measured with Western blotting analysis using polyclonal Abs raised against ADMA (Active Motif Inc.) and AGEs as described previously. ${ }^{19,25}$ All animal procedures were conducted according to the guidelines provided by the Kurume University Institutional Animal Care and Use Committee under an approved protocol.

\section{Histopathologic Examinations}

The kidneys were fixed in $4 \%$ paraformaldehyde and embedded in paraffin, sectioned at $4-\mu \mathrm{m}$ intervals, and mounted on glass slides. The sections were stained with H\&E and Masson's trichrome for light microscopic analysis. Glomerular area delimited by the internal edge of the Bowman's capsule was measured using ImageJ software. The intensity of Masson's trichrome staining in the glomeruli and tubulointerstitium were quantitatively analyzed by Optimas image analysis software version 6.57 (Media Cybernetics, Silver Spring, MD).

\section{Immunohistochemical Analysis}

The kidney sections were incubated in $0.3 \%$ hydrogen peroxide methanol for 30 minutes to block endogenous peroxidase activity and incubated overnight at $4{ }^{\circ} \mathrm{C}$ with F4/80 Ab directed specially against macrophages (Santa Cruz Biotechnology Inc.). Then the reactions were visualized with an avidin-biotin-alkaline phosphatase kit (Vectastain ABC-AP; Vector Laboratories, Inc., Burlingame, CA). Ten 

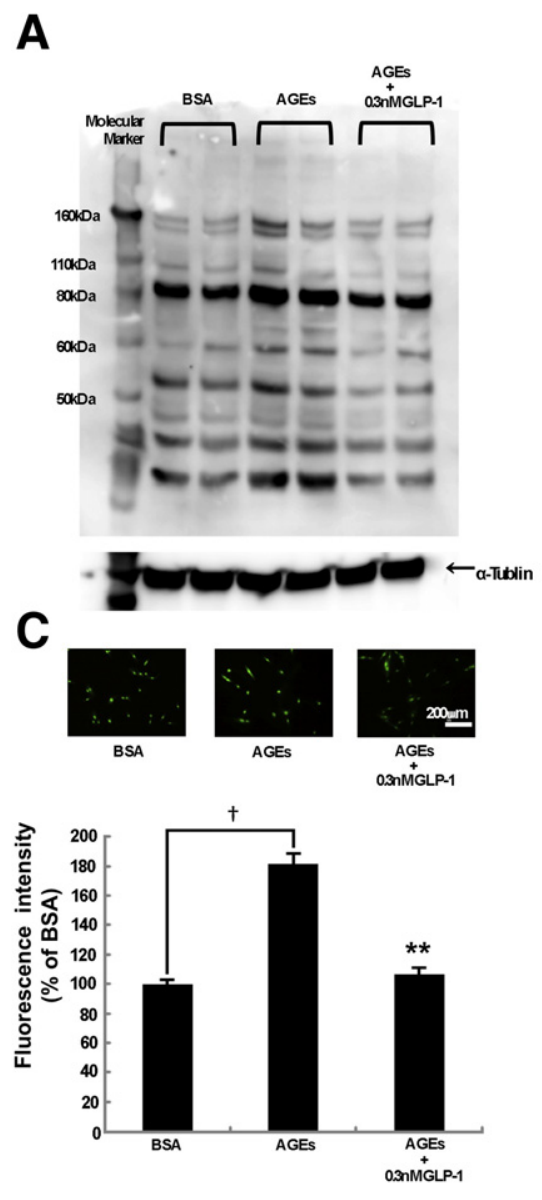

\section{B}

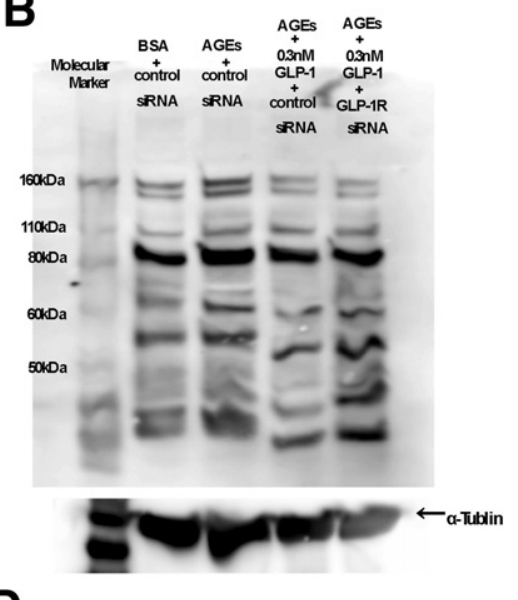

D

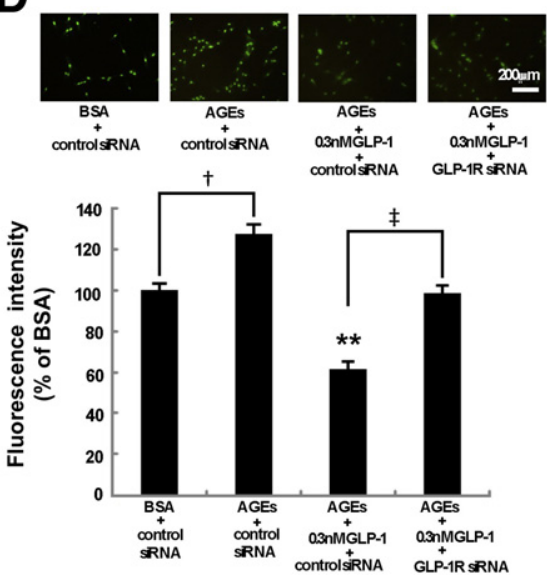

Figure 3 Effects of GLP-1 or GLP-1R siRNA on ADMA levels in tubular cells. Transfected or nontransfected cells were treated with or without 100 $\mu \mathrm{g} / \mathrm{mL}$ of AGE-BSA or nonglycated BSA in the presence or absence of $0.3 \mathrm{nmol} / \mathrm{L}$ GLP-1 for 4 hours. Then ADMA levels were measured by Western blotting ( $\mathbf{A}$ and $\mathbf{B}$ ) and immunostaining analyses ( $\mathbf{C}$ and $\mathbf{D})$. A and $\mathbf{B}$ : Representative bands of Western blots. Three independent experiments obtained the same results. C and D: Each upper panel shows the representative microphotographs. Each lower panel shows the quantitative data of intensity of ADMA immunostaining. ${ }^{*} P<0.01$ compared with the value with AGEs (C) or AGEs plus control siRNA (D). ${ }^{\dagger} P<0.01$ compared with the value with BSA (C) or BSA plus control siRNA (D). ${ }^{\ddagger} P<0.01$ compared with the value with AGEs plus $0.3 \mathrm{nmol} / \mathrm{L} \mathrm{GLP}-1$ plus control siRNA $(n=6$ per group). different fields in each sample were chosen, and the number of $\mathrm{F} 4 / 80^{+}$cells per glomerulus was counted.

\section{Statistical Analysis}

Unless otherwise indicated, all values were presented as mean \pm SE. One-way analysis of variance followed by the Scheffé F test was performed for statistical comparisons; $P<0.05$ was considered significant.

\section{Results}

GLP-1 Inhibits PRMT-1 Gene Expression via ROS Generation in Tubular Cells through the Interaction with RAGE

Engagement of RAGE with AGEs activates its downstream signaling via ROS generation in various types of cells. ${ }^{4-8}$ Therefore, we first examined the effect of GLP-1 on

Table 1 Clinical Characteristics of the Study Animals

\begin{tabular}{lcccc}
\hline Characteristic & Control $(n=5)$ & $\begin{array}{l}\text { STZ and } \\
\text { vehicle }(n=3)\end{array}$ & $\begin{array}{l}\text { STZ and low-dose } \\
\text { exendin-4 }(n=4)\end{array}$ & $\begin{array}{c}\text { STZ and high-dose } \\
\text { exendin-4 }(n=4)\end{array}$ \\
\hline Body weight $(\mathrm{g})$ & $292 \pm 7$ & $211 \pm 41^{*}$ & $198 \pm 14^{\dagger}$ & $233 \pm 21^{*}$ \\
Heart rate (beats per minute) & $414 \pm 14$ & $317 \pm 38^{*}$ & $329 \pm 17^{\dagger}$ & $372 \pm 21$ \\
Mean blood pressure (mmHg) & $91 \pm 3$ & $72 \pm 5^{\dagger}$ & $90 \pm 7$ & $92 \pm 7$ \\
Blood glucose (mg/dL) & $121 \pm 4$ & $585 \pm 94^{\dagger}$ & $720 \pm 73^{\dagger}$ & $478 \pm 34^{\dagger}$ \\
Total cholesterol (mg/dL) & $62 \pm 4$ & $144 \pm 1$ & $88 \pm 21$ & $56 \pm 14$ \\
HDL-C (mg/dL) & $42 \pm 2$ & $48 \pm 17$ & $52 \pm 7$ & $39 \pm 11$ \\
Aspartate aminotransferase & $68 \pm 6$ & $216 \pm 12$ & $204 \pm 13^{*}$ & $120 \pm 32$ \\
Alanine aminotransferase & $29 \pm 3$ & $102 \pm 22^{\dagger}$ & $135 \pm 19^{\dagger}$ & $77 \pm 19^{*}$ \\
Creatinine & $0.21 \pm 0.01$ & $0.2 \pm 0.06$ & $0.2 \pm 0.04$ & $0.16 \pm 0.02$ \\
\hline
\end{tabular}

Data are presented as mean \pm SE.

${ }^{*} P<0.05$ compared with control.

${ }^{\dagger} P<0.01$ compared with control.

HDL-C, high-density lipoprotein cholesterol. 

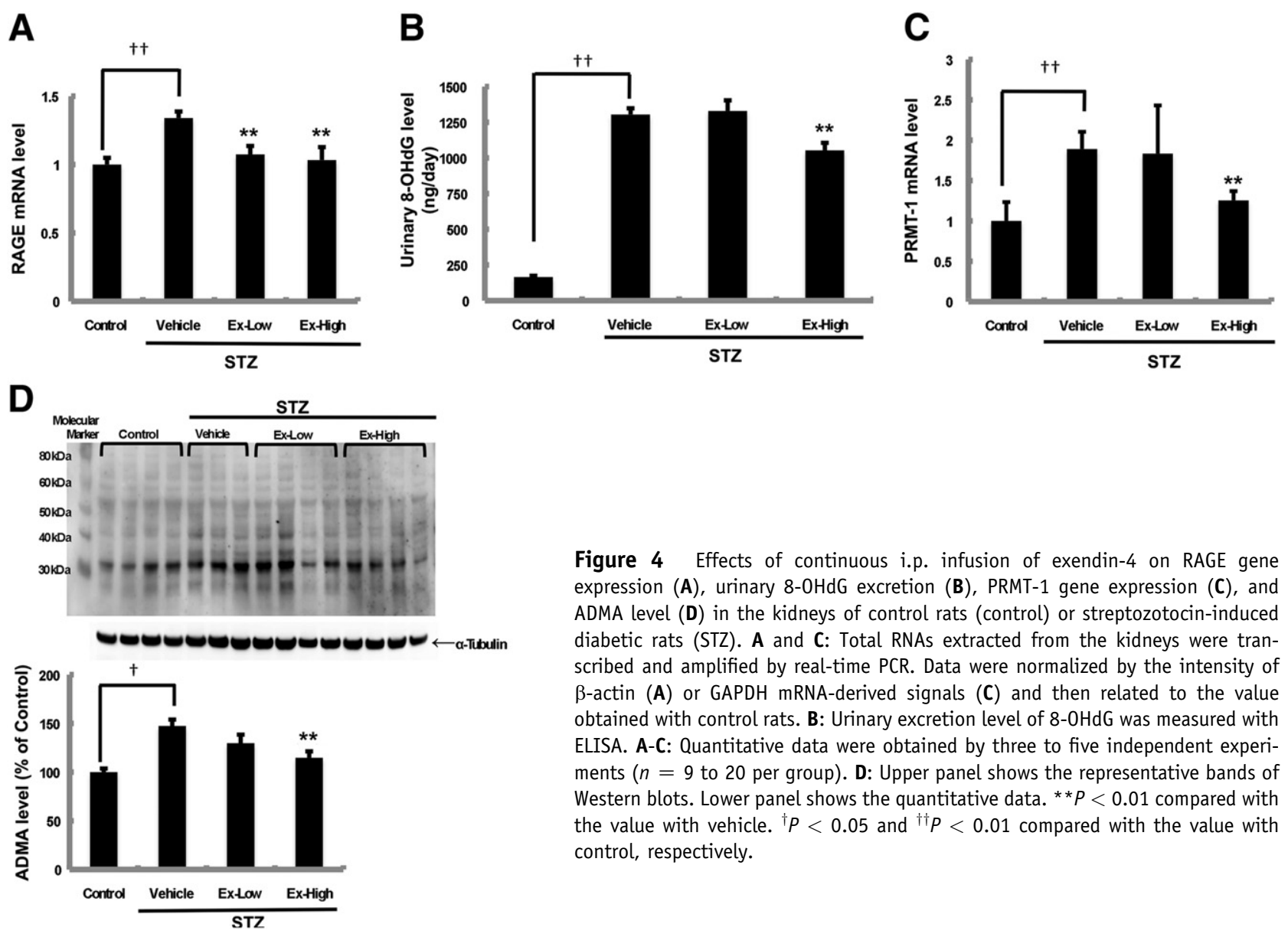

\begin{abstract}
Figure 4 Effects of continuous i.p. infusion of exendin-4 on RAGE gene expression (A), urinary 8-0HdG excretion (B), PRMT-1 gene expression (C), and ADMA level (D) in the kidneys of control rats (control) or streptozotocin-induced diabetic rats (STZ). A and C: Total RNAs extracted from the kidneys were transcribed and amplified by real-time PCR. Data were normalized by the intensity of $\beta$-actin (A) or GAPDH mRNA-derived signals (C) and then related to the value obtained with control rats. B: Urinary excretion level of 8-0HdG was measured with ELISA. A-C: Quantitative data were obtained by three to five independent experiments ( $n=9$ to 20 per group). D: Upper panel shows the representative bands of Western blots. Lower panel shows the quantitative data. ${ }^{* *} p<0.01$ compared with the value with vehicle. ${ }^{\dagger} P<0.05$ and ${ }^{\dagger \dagger} P<0.01$ compared with the value with control, respectively.
\end{abstract}

RAGE gene expression and ROS generation in tubular cells. As shown in Figure 1, A and B, $0.3 \mathrm{nmol} / \mathrm{L}$ GLP-1 significantly inhibited the AGE-induced increase in RAGE mRNA levels and superoxide generation in tubular cells. Furthermore, AGEs up-regulated PRMT-1 mRNA levels in tubular cells, which were blocked by the treatment with 0.3 nmol/L GLP-1, neutralizing RAGE-Ab, or an anti-oxidant NAC (Figure 1, C and D).

\section{GLP-1 Inhibits PRMT-1 mRNA Level in Tubular Cells via the Activation of GLP-1R-cAMP System}

Because of the ambiguity as to the location of GLP-1R in human kidney, we examined whether proximal tubular cells expressed GLP-1R. As shown in Figure 2A, Western blotting analysis revealed a single band with a molecular mass of $56 \mathrm{kDa}$, corresponding to the GLP-1R seen in tubular cells. We also confirmed that GLP-1R was barely detectable in tubular cells exposed to siRNA molecules specific for human GLP-1R (Figure 2A).

We next examined the involvement of GLP-1R in GLP-1 actions on tubular cells. For this, we investigated the effects of siRNAs raised against GLP-1R on RAGE gene expression, superoxide generation, and PRMT-1 mRNA level in tubular cells. Control siRNA did not affect the effects of
AGEs on tubular cells; AGEs significantly increased RAGE and PRMT-1 mRNA levels and ROS generation in control siRNA-treated cells (Figure 2, B-D). GLP-1 decreased RAGE gene expression, ROS generation, and PRMT-1 mRNA level in AGEs plus control siRNA-exposed tubular cells, all of which were significantly blocked by the treatment with GLP-1R siRNA transfection (Figure 2, B-D). Because the actions of the GLP-1R are mediated by cAMP production, ${ }^{27}$ we further studied the effects of an analog of cAMP, 8-Br-cAMP on RAGE, and PRMT-1 gene expression in tubular cells. As shown in Figure 2, E and F, 8-BrcAMP significantly reduced RAGE and PRMT-1 mRNA levels in tubular cells.

\section{GLP-1-GLP-1R Interaction Decreases ADMA Level in AGE-Exposed Tubular Cells}

We investigated whether GLP-1 could reduce ADMA level in AGE-exposed tubular cells. As shown in Figure 3, A-D, Western blotting and immunostaining analyses revealed that AGEs significantly increased ADMA-modified protein levels in tubular cells, which was prevented by the treatment with $0.3 \mathrm{nmol} / \mathrm{L}$ GLP-1. Furthermore, GLP-1R siRNA blocked the suppressive effects of GLP-1 on ADMA levels 

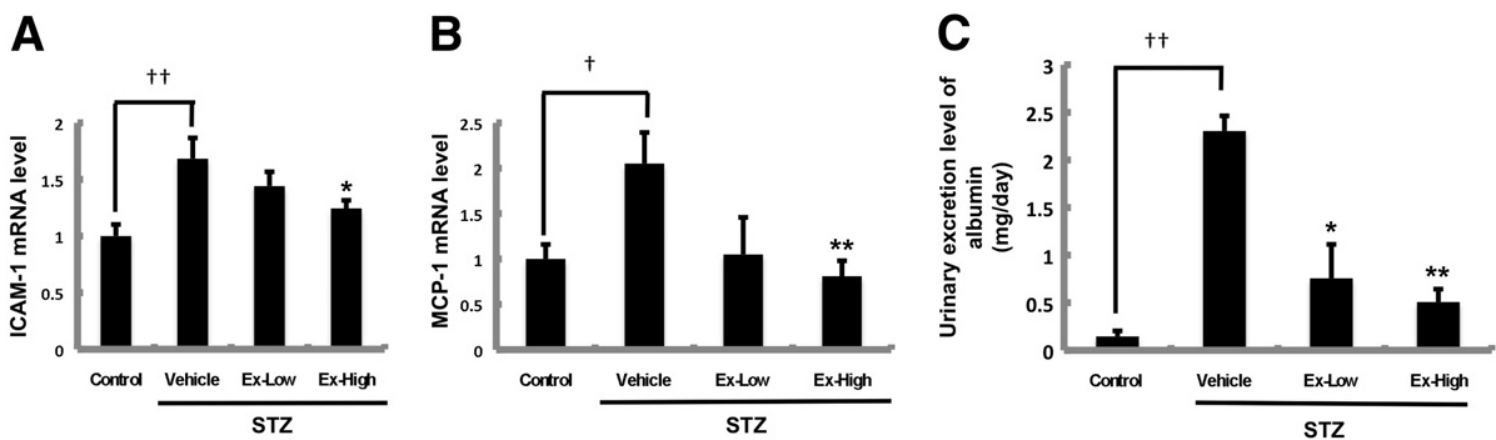

Figure 5 Effects of continuous i.p. infusion of exendin-4 on renal ICAM-1 (A), MCP-1 gene expression (B), and urinary excretion level of albumin (C) in control rats (control) or streptozotocin-induced diabetic rats (STZ). A and B: Total RNAs extracted from the kidneys were transcribed and amplified by real-time PCR. Data were normalized by the intensity of $18 \mathrm{~S}$ mRNA-derived signals and then related to the value obtained with control rats. C: Urinary excretion level of 8-OHdG was measured with ELISA. ${ }^{*} P<0.05$ and ${ }^{*} * P<0.01$ compared with the value with vehicle, respectively; ${ }^{\dagger} P<0.05$ and ${ }^{\dagger \dagger} P<0.01$ compared with the value with control, respectively. Quantitative data were obtained by three independent experiments ( $n=9$ to 12 per group).

in AGEs plus control siRNA-exposed tubular cells (Figure 3, $\mathrm{A}-\mathrm{D})$.

\section{Continuous I.P. Infusion of the GLP-1 Analog}

Exendin-4 Reduces Rage and Prmt-1 Gene Expression and ADMA Levels in the Kidney and Decreases Urinary Excretion Levels of 8-0HdG in Experimental Diabetic Nephropathy

Because tubular cells are major constituents of kidney, ${ }^{28}$ we next studied the effects of exendin-4 on streptozotocininduced early phase of diabetic nephropathy. As indicated in Table 1, compared with the control rats, body weight was heavier and heart rate, blood glucose, and alanine aminotransferase levels were higher, while mean blood pressure was lower in diabetic rats. Although low or high doses of exendin-4 infusion did not affect these parameters in diabetic rats, a high dose of exendin- 4 treatment significantly inhibited renal Rage and Prmt-1 gene expression, decreased urinary excretion level of $8-\mathrm{OHdG}$, and reduced ADMAmodified protein levels in the kidney in streptozotocininduced diabetic animals (Figure 4, A-D).

\section{Continuous I.P. Infusion of Exendin-4 Inhibits Inflammatory Reactions in the Kidney and Reduces Urinary Excretion Level of Albumin in Streptozotocin- Induced Diabetic Rats}

As shown in Figure 5, A-C, compared with the control rats, renal gene expression of ICAM-1 and MCP-1 and urinary
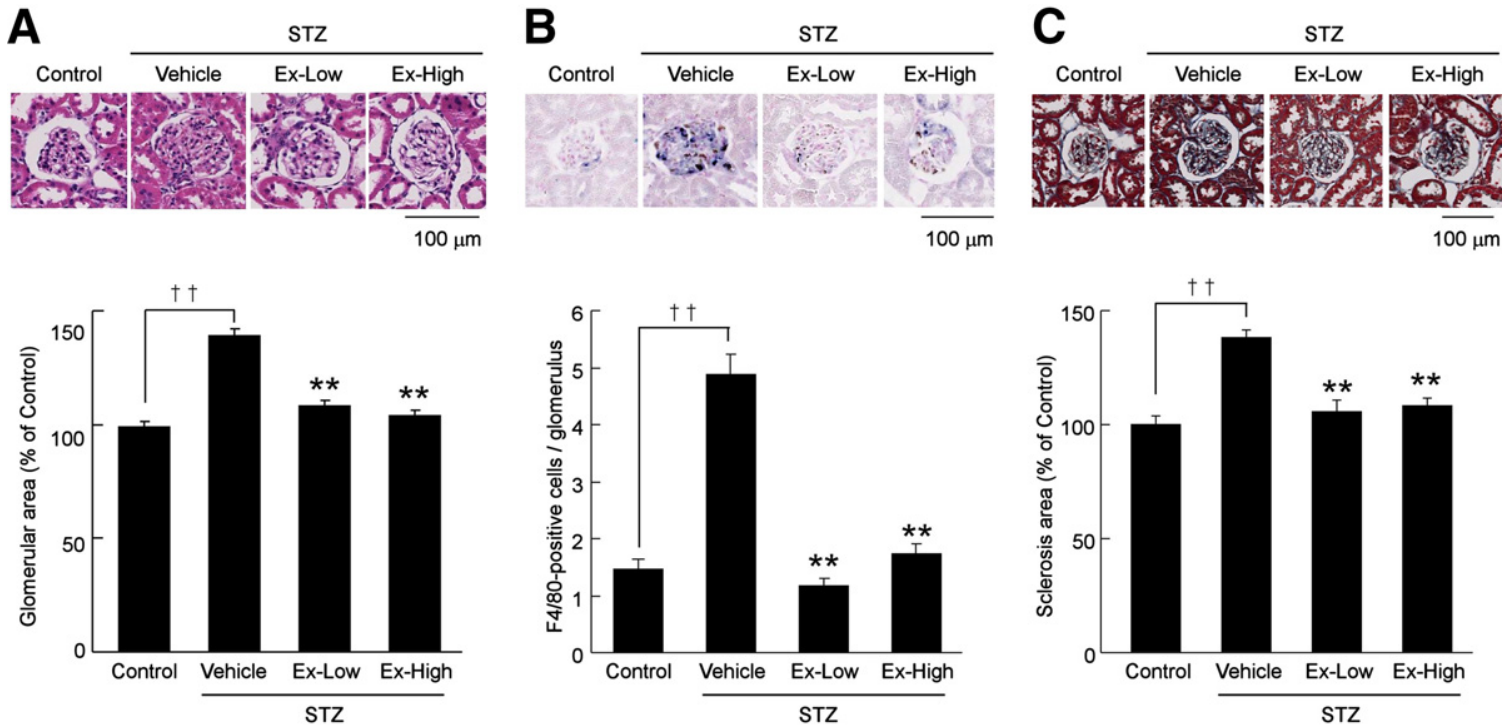

Figure 6 Effects of continuous i.p. infusion of exendin-4 on glomerular area (A), macrophage infiltration in the glomeruli (B), and glomerular and tubulointerstitial fibrosis (C) in control rats (control) or streptozotocin-induced diabetic rats (STZ). The kidney sections were stained with H\&E (A) and Masson's trichrome (C) for light microscopic analysis. Glomerular area was measured using ImageJ software, and the intensity of Masson's trichrome staining in the glomeruli and tubulointerstitium was quantitatively analyzed by image analysis software. B: The number of $\mathrm{F} 4 / 80^{+}$cells per glomerulus was counted. ${ }^{* *} P<0.01$ compared with the value with vehicle; ${ }^{\dagger \dagger} P<0.01$ compared with the value with control. Ten different fields in each sample were evaluated $(n=40$ per group). 
excretion level of albumin were increased in diabetic rats, all of which were significantly prevented by the treatment of high dose of exendin-4.

\section{Continuous I.P. Infusion of Exendin-4 Inhibits} Glomerular Hypertrophy and Macrophage Infiltration in the Glomeruli and Glomerular and Tubulointerstitial Fibrosis in Streptozotocin-Induced Diabetic Rats

As shown in Figure 6, A-C, compared with the control rats, glomerular area was increased, and macrophage infiltration in the glomeruli and glomerular and tubulointerstitial fibrosis were enhanced in diabetic rats, all of which were significantly prevented by the treatment of low or high dose of exendin-4. We also confirmed that AGE-modified protein levels were significantly increased in the kidney of 2-weekold diabetic rats (Figure 7).

\section{Discussion}

In this study, we demonstrated for the first time that GLP-1 inhibited the AGE-induced RAGE gene expression, ROS generation, and PRMT-1 mRNA level and subsequently decreased ADMA level in cultured human proximal tubular cells. In addition, RAGE-Ab or an antioxidant NAC was found to inhibit the AGE-induced tubular cell gene expression of PRMT-1, a rate-limiting enzyme for ADMA generation. ${ }^{15-17}$ We also found here that continuous i.p. infusion of the GLP-1 analog exendin-4 (1.5 $\mu \mathrm{g} / \mathrm{kg} / \mathrm{h})$ inhibited renal RAGE gene expression, reduced urinary excretion level of 8-OHdG (an oxidative stress marker), ${ }^{28}$ improved histologic changes, and decreased PRMT-1 mRNA level and ADMA generation in
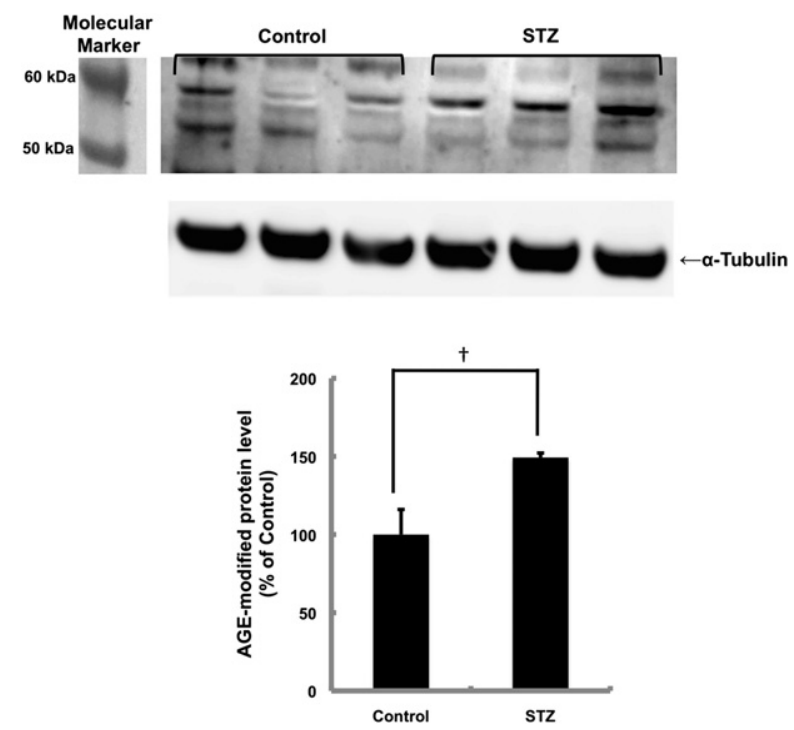

Figure 7 AGE-modified protein levels in kidneys of control rats (control) or streptozotocin-induced diabetic rats (STZ). Upper panel shows the representative bands of Western blots. Lower panel shows the quantitative data. Data were normalized by the intensity of $\alpha$-tubulin bands and then related to the value obtained with the control. ${ }^{\dagger} P<0.05$ compared to the value with control. the kidney in streptozotocin-induced diabetic rats, although it did not affect blood glucose or lipid levels. Given that RAGE is a major receptor for AGEs that mediates the downstream signaling and that ROS generation works as a second messenger of RAGE, ${ }^{4-8}$ the present study suggests that RAGE gene suppression in tubular cells could be a central mechanism by which GLP-1 inhibits the ADMA level in the kidney in the early phase of experimental diabetic nephropathy. There is accumulating evidence that ADMA, an endogenous inhibitor of NO synthase, plays a role in endothelial dysfunction, cardiovascular remodeling, proteinuria, and progression of renal damage in high-risk patients for cardiovascular disease, such as those complicated with diabetes and chronic kidney disease. ${ }^{15-17,29-35}$ Therefore, suppression of the AGERAGE-induced ADMA generation by GLP-1 may be a novel therapeutic target for vascular complications in diabetes.

In the present study, we found that GLP-1R was expressed in proximal tubular epithelial cells and that GLP-1R siRNAs but not control siRNAs inhibited the GLP-1-induced downregulation of RAGE mRNA level. Furthermore, effects of GLP-1 on superoxide generation, PRMT-1 gene expression, and ADMA generation in AGE-exposed tubular cells were blocked by the treatment with siRNA molecules specific for human GLP-1R. In addition, an analog of cAMP, 8-Br-cAMP, mimicked the effects of GLP-1 on RAGE and PRMT-1 expression in tubular cells. Because GLP-1R mainly mediates the biological actions of GLP-1 on pancreatic cells via the cAMP pathway, ${ }^{10,27}$ our present results suggest that the ADMA-lowering actions of GLP-1 on tubular cells are partly mediated by cAMP pathway via the interaction with GLP-1R. We have previously shown that AGEs decrease intracellular cAMP levels in human cultured endothelial cells and that cAMP agonists, such as dibutyryl cAMP, block the AGERAGE signaling pathway. ${ }^{36}$ Moreover, beraprost sodium, an active prostacyclin analog with cAMP-elevating activity, has been reported to down-regulate RAGE mRNA levels in AGEexposed pericytes. ${ }^{37}$ These findings suggest that the GLP1-GLP-1R-cAMP axis may suppress RAGE expression and its downstream pathway in AGE-exposed tubular cells.

We have previously found that RAGE-Ab completely blocks the AGE-induced up-regulation of RAGE mRNA level in tubular cells. ${ }^{28}$ Furthermore, AGEs increased RAGE mRNA levels in tubular cells via NADPH oxidase-mediated ROS generation. ${ }^{20,28,38}$ Therefore, it is conceivable that the AGE-RAGE-induced NADPH oxidase-mediated ROS generation could further stimulate RAGE gene expression, thereby forming a positive feedback loop between RAGE induction and ROS generation in AGE-exposed tubular cells. Because the GLP-1 analog liraglutide has been recently found to inhibit oxidative stress generation in streptozotocin-induced diabetic rats via cAMP-mediated inhibition of renal NADPH oxidase, ${ }^{39}$ blockade of NADPH oxidase-induced ROS generation by cAMP may be a molecular mechanism by which GLP-1 or exendin-4 down-regulate RAGE expression in AGEexposed tubular cells or diabetic kidney, respectively. 
In this study, we found that RAGE-Ab or NAC inhibited up-regulation of PRMT-1 mRNA levels in tubular cells. Lysophosphatidylcholine, a major component of oxidized lowdensity lipoprotein, was reported to increase intracellular ROS production, PRMT-1 expression, and ADMA level in endothelial cells, all of which were blocked by the treatment with an inhibitor of NADPH oxidase. ${ }^{40}$ Low-density lipoprotein was also found to enhance gene expression of PRMT-1 and subsequently stimulate ADMA generation in endothelial cells, which was blocked by an antioxidant pyrrolidine dithiocarbamate. ${ }^{41}$ Therefore, reduction of PRMT-1 mRNA levels by GLP-1 may be ascribed to its antioxidative properties.

In the present study, we found that continuous i.p. infusion of exendin- 4 decreased renal expression of ICAM- 1 and monocyte chemotactic protein 1 (MCP-1), reduced albuminuria, prevented glomerular hypertrophy and macrophage infiltration in the glomeruli, and blocked glomerular and tubulointerstitial fibrosis in streptozotocin-induced diabetic rats. ADMA could injure glomerular filtration barrier and, therefore, enhance albumin permeability of isolated glomeruli. ${ }^{42}$ Reduction of renal ADMA level by exendin-4 may partly account for its albuminuria-lowering action in diabetic rats. Increased expression of ICAM-1 and MCP-1 expression is associated with monocyte infiltration into both mesangial and tubulointerstitial lesions in diabetic nephropathy. ${ }^{43-45}$ Urinary MCP-1/ creatinine and ICAM-1/creatinine ratios in type 2 diabetic patients with microalbuminuria were much higher than those in healthy controls, and intensive insulin treatment significantly decreased urinary MCP-1, ICAM-1, and albumin excretions. ${ }^{46}$ Furthermore, ADMA has been found to promote monocyte adhesion by inducing MCP1 and ICAM-1 expression in endothelial cells. ${ }^{47,48}$ Therefore, exendin-4 may reduce albuminuria and histopathologic damage in diabetic rats through its anti-inflammatory properties via suppression of ADMA.

In vitro modified AGEs were prepared by incubating BSA with glyceraldehyde for 1 week; this process produces relatively highly modified proteins compared with those in vivo. However, it is unlikely that extensively modified, unphysiologic AGEs that were formed under the in vitro conditions may exert nonspecific and toxic effects on tubular cell damage for the following reasons: we have previously found that immunologic epitope of glyceraldehyde-modified AGEs was actually present in the serum of diabetic patients, and that the concentration (100 $\mu \mathrm{g} / \mathrm{mL}$ ) of in vitro prepared AGEs used here was comparable with those of the in vivo diabetic situation, and this type of AGE mimics the biological effects of AGE-rich serum fractions purified from diabetic patients receiving

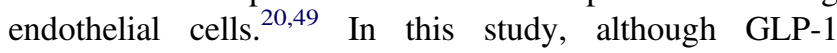
modestly restored the AGE-induced decrease in gene expression of DDAH-1, a key enzyme that mainly degrades ADMA in tubular cells, no significant difference was found in DDAH-1 gene expression levels among control, diabetic, and exendin-4-treated diabetic rats (data not shown). Therefore, the involvement of DDAH-1 in exendin4-induced reduction of ADMA in the diabetic kidney remains unclear. Because the experimental animal model does not completely mimic human diabetic nephropathy, further study is needed to clarify whether GLP-1R agonist treatment could be a therapeutic strategy for the treatment of diabetic nephropathy in humans.

\section{References}

1. Vlassara H, Bucala R: Recent progress in advanced glycation and diabetic vascular disease: role of advanced glycation end product receptors. Diabetes Suppl 1996, 3:S65-S66

2. Brownlee M, Cerami A, Vlassara A: Advanced glycosylation end products in tissue and the biochemical basis of diabetic complications. N Engl J Med 1998, 318:1315-1321

3. Rahbar S: Novel inhibitors of glycation and AGE formation. Cell Biochem Biophys 2007, 48:147-157

4. Yamamoto Y, Kato I, Doi T, Yonekura H, Ohashi S, Takeuchi M, Watanabe T, Yamagishi S, Sakurai S, Takasawa S, Okamoto $\mathrm{H}$ Yamamoto H: Development and prevention of advanced diabetic nephropathy in RAGE-overexpressing mice. J Clin Invest 2001, 108 : 261-268

5. Wendt TM, Tanji N, Guo J, Kislinger TR, Qu W, Lu Y, Bucciarelli LG, Rong LL, Moser B, Markowitz GS, Stein G, Bierhaus A, Liliensiek B, Arnold B, Nawroth PP, Stern DM, D'Agati VD, Schmidt AM: RAGE drives the development of glomerulosclerosis and implicates podocyte activation in the pathogenesis of diabetic nephropathy. Am J Pathol 2003, 162:1123-1137

6. Reiniger N, Lau K, McCalla D, Eby B, Cheng B, Lu Y, Qu W, Quadri N, Ananthakrishnan R, Furmansky M, Rosario R, Song F, Rai V, Weinberg A, Friedman R, Ramasamy R, D'Agati V, Schmidt AM: Deletion of the receptor for advanced glycation end products reduces glomerulosclerosis and preserves renal function in the diabetic OVE26 mouse. Diabetes 2010, 59:2043-2054

7. Yamagishi S, Imaizumi T: Diabetic vascular complications: pathophysiology, biochemical basis and potential therapeutic strategy. Curr Pharm Des 2005, 11:2279-2299

8. Yamagishi S, Matsui T: Advanced glycation end products, oxidative stress and diabetic nephropathy. Oxid Med Cell Longev 2010, 3:101-108

9. Kim W, Egan JM: The role of incretins in glucose homeostasis and diabetes treatment. Pharmacol Rev 2008, 60:470-512

10. Winzell MS, Ahrén B: G-protein-coupled receptors and islet functionimplications for treatment of type 2 diabetes. Pharmacol Rev 2007 116:437-448

11. Chilton R, Wyatt J, Nandish S, Oliveros R, Lujan M: Cardiovascular comorbidities of type 2 diabetes mellitus: defining the potential of glucagonlike peptide-1-based therapies. Am J Med 2011, 124(1 Suppl): S35-S53

12. Anagnostis P, Athyros VG, Adamidou F, Panagiotou A, Kita M, Karagiannis A, Mikhailidis DP: Glucagon-like peptide-1-based therapies and cardiovascular disease: looking beyond glycaemic control. Diabetes Obes Metab 2011, 13:302-312

13. Baylis C: Nitric oxide deficiency in chronic kidney disease. Am J Physiol Renal Physiol 2008, 294:F1-F9

14. Yamagishi S, Matsui T: Nitric oxide, a janus-faced therapeutic target for diabetic microangiopathy: friend or foe? Pharmacol Res 2011, 64:187-194

15. Yamagishi S, Ueda S, Nakamura K, Matsui T, Okuda S: Role of asymmetric dimethylarginine (ADMA) in diabetic vascular complications. Curr Pharm Des 2008, 14:2613-2618

16. Böger RH, Bode-Böger SM, Frölich JC: The L-arginine-nitric oxide pathway: role in atherosclerosis and therapeutic implications. Atherosclerosis $1996,127: 1-11$

17. Tarnow L, Hovind P, Teerlink T, Stehouwer CD, Parving $\mathrm{HH}$ Elevated plasma asymmetric dimethylarginine as a marker of cardiovascular morbidity in early diabetic nephropathy in type 1 diabetes. Diabetes Care 2004, 27:765-769 
18. Nakamura T, Sato E, Fujiwara N, Kawagoe Y, Ueda Y, Suzuki T, Yamada S, Takeuchi M, Fukami K, Ueda S, Adachi H, Matsui T, Okuda S, Yamagishi S: Positive association of serum levels of advanced glycation end products and high mobility group box-1 with asymmetric dimethylarginine in nondiabetic chronic kidney disease patients. Metabolism 2009, 58:1624-1628

19. Takeuchi M, Makita Z, Bucala R, Suzuki T, Koike T, Kameda Y: Immunological evidence that non-carboxymethyllysine advanced glycation end-products are produced from short chain sugars and dicarbonyl compounds in vivo. Mol Med 2000, 6:114-125

20. Yamagishi S, Nakamura K, Matsui T, Inagaki Y, Takenaka K, Jinnouchi Y, Yoshida Y, Matsuura T, Narama I, Motomiya Y, Takeuchi M, Inoue H, Yoshimura A, Bucala R, Imaizumi T: Pigment epithelium-derived factor inhibits advanced glycation end productinduced retinal vascular hyperpermeability by blocking reactive oxygen species-mediated vascular endothelial growth factor expression. J Biol Chem 2006, 281:20213-20220

21. Yamagishi S, Yonekura H, Yamamoto Y, Katsuno K, Sato F, Mita I, Ooka H, Satozawa N, Kawakami T, Nomura M, Yamamoto H: Advanced glycation end products-driven angiogenesis in vitro. Induction of the growth and tube formation of human microvascular endothelial cells through autocrine vascular endothelial growth factor. J Biol Chem 1997, 272:8723-8730

22. Sasaki N, Takeuchi M, Chowei H, Kikuchi S, Hayashi Y, Nakano N, Ikeda H, Yamagishi S, Kitamoto T, Saito T, Makita Z: Advanced glycation end products (AGE) and their receptor (RAGE) in the brain of patients with Creutzfeldt-Jakob disease with prion plaques. Neurosci Lett 2002, 326:117-120

23. Yamagishi S, Inagaki Y, Okamoto T, Amano S, Koga K, Takeuchi M: Advanced glycation end products inhibit de novo protein synthesis and induce TGF-beta overexpression in proximal tubular cells. Kidney Int 2003, 63:464-473

24. Ishibashi Y, Matsui T, Takeuchi M, Yamagishi S: Glucagon-like peptide-1 (GLP-1) inhibits advanced glycation end product (AGE)induced up-regulation of VCAM-1 mRNA levels in endothelial cells by suppressing AGE receptor (RAGE) expression. Biochem Biophys Res Commun 2010, 391:1405-1408

25. Yoshida $T$, Yamagishi $S$, Nakamura $K$, Matsui $T$, Imaizumi $T$, Takeuchi M, Koga H, Ueno T, Sata M: Telmisartan inhibits AGEinduced C-reactive protein production through downregulation of the receptor for AGE via peroxisome proliferator-activated receptorgamma activation. Diabetologia 2006, 49:3094-3099

26. Fujimura $T$, Yamagishi S, Ueda S, Fukami K, Shibata R, Matsumoto Y, Kaida Y, Hayashida A, Koike K, Matsui T, Nakamura K, Okuda S: Administration of pigment epithelium-derived factor (PEDF) reduces proteinuria by suppressing decreased nephrin and increased VEGF expression in the glomeruli of adriamycininjected rats. Nephrol Dial Transplant 2009, 24:1397-1406

27. Kieffer TJ, Habener JF: The glucagon-like peptides. Endocr Rev 1999, 20:876-913

28. Maeda S, Matsui T, Takeuchi M, Yoshida Y, Yamakawa R, Fukami K, Yamagishi S: Pigment epithelium-derived factor (PEDF) inhibits proximal tubular cell injury in early diabetic nephropathy by suppressing advanced glycation end products (AGEs)-receptor (RAGE) axis. Pharmacol Res 2011, 63:241-248

29. Matsui T, Nishino Y, Takeuchi M, Yamagishi S: Vildagliptin blocks vascular injury in thoracic aorta of diabetic rats by suppressing advanced glycation end product-receptor axis. Pharmacol Res 2011, 63:383-388

30. Ueda S, Yamagishi S, Kaida Y, Okuda S: Asymmetric dimethylarginine may be a missing link between cardiovascular disease and chronic kidney disease. Nephrology (Carlton) 2007, 12:582-590

31. Zoccali C, Benedetto FA, Maas R, Mallamaci F, Tripepi G, Malatino LS, Böger R, CREED Investigators: Asymmetric dimethylarginine, C-reactive protein, and carotid intima-media thickness in end-stage renal disease. J Am Soc Nephrol 2002, 13:490-496

32. Zoccali C, Mallamaci F, Maas R, Benedetto FA, Tripepi G, Malatino LS, Cataliotti A, Bellanuova I, Böger R, CREED Investigators: Left ventricular hypertrophy, cardiac remodeling and asymmetric dimethylarginine in hemodialysis patients. Kidney Int 2002, 62:339-345

33. Cross JM, Donald A, Vallance PJ, Deanfield JE, Woolfson RG, MacAllister RJ: hemodialysis improves endothelial function in humans. Nephrol Dial Transplant 2001, 16:1823-1829

34. Caglar K, Yilmaz MI, Sonmez A, Cakir E, Kaya A, Acikel C, Eyileten T, Yenicesu M, Oguz Y, Bilgi C, Oktenli C, Vural A, Zoccali C: ADMA, proteinuria, and insulin resistance in non-diabetic stage I chronic kidney disease. Kidney Int 2006, 70:781-787

35. Fliser D, Kronenberg F, Kielstein JT, Morath C, Bode-Boger SM, Haller H, Ritz E: Asymmetric dimethylarginine and progression of chronic kidney disease: the mild to moderate kidney disease study. J Am Soc Nephrol 2005, 16:2456-2461

36. Yamagishi S, Fujimori H, Yonekura H, Yamamoto Y, Yamamoto $\mathrm{H}$ : Advanced glycation endproducts inhibit prostacyclin production and induce plasminogen activator inhibitor-1 in human microvascular endothelial cells. Diabetologia 1998, 41:1435-1441

37. Yamagishi S, Amano S, Inagaki Y, Okamoto T, Takeuchi M, Makita Z: Beraprost sodium, a prostaglandin I2 analogue, protects against advanced gycation end products-induced injury in cultured retinal pericytes. Mol Med 2002, 8:546-550

38. Matsui T, Yamagishi S, Takeuchi M, Ueda S, Fukami K, Okuda S: Irbesartan inhibits advanced glycation end product (AGE)-induced proximal tubular cell injury in vitro by suppressing receptor for AGEs (RAGE) expression. Pharmacol Res 2010, 61:34-39

39. Hendarto $\mathrm{H}$, Inoguchi $\mathrm{T}$, Maeda $\mathrm{Y}$, Ikeda $\mathrm{N}$, Zheng J, Takei R, Yokomizo H, Hirata E, Sonoda N, Takayanagi R: GLP-1 analog liraglutide protects against oxidative stress and albuminuria in streptozotocin-induced diabetic rats via protein kinase A-mediated inhibition of renal NAD(P)H oxidases. Metabolism 2012, 61(10):1422-1434

40. Jia SJ, Jiang DJ, Hu CP, Zhang XH, Deng HW, Li YJ: Lysophosphatidylcholine-induced elevation of asymmetric dimethylarginine level by the NADPH oxidase pathway in endothelial cells. Vascul Pharmacol 2006, 44:143-148

41. Böger RH, Sydow K, Borlak J, Thum T, Lenzen H, Schubert B, Tsikas D, Bode-Böger SM: LDL cholesterol upregulates synthesis of asymmetrical dimethylarginine in human endothelial cells: involvement of S-adenosylmethionine-dependent methyltransferases. Circ Res 2000, 87:99-105

42. Sharma M, McCarthy ET, Savin VJ, Lianos EA: Nitric oxide preserves the glomerular protein permeability barrier by antagonizing superoxide. Kidney Int 2005, 68:2735-2744

43. Banba N, Nakamura T, Matsumura M, Kuroda H, Hattori Y, Ksai K Possible relationship of monocyte chemoattractant protein-1 with diabetic nephropathy. Kidney Int 2000, 58:684-960

44. Sugimoto H, Shikata K, Hirata K, Akiyama K, Matsuda M, Kushiro M, Shikata Y, Miyatake N, Miyasaka M, Makino H: Increased expression of intercellular adhesion molecule-1 (ICAM-1) in diabetic rat glomeruli: glomerular hyperfiltration is a potential mechanism of ICAM-1 upregulation. Diabetes 1997, 46:2075-2201

45. Chow FY, Nikolic-Paterson DJ, Ozols E, Atkins RC, Tesch GH: Intercellular adhesion molecule-1 deficiency is protective against nephropathy in type 2 diabetic db/db mice. J Am Soc Nephrol 2005, 16:1711-1722

46. Ye SD, Zheng M, Zhao LL, Qian Y, Yao XM, Ren A, Li SM, Jing CY: Intensive insulin therapy decreases urinary MCP-1 and ICAM-1 excretions in incipient diabetic nephropathy. Eur J Clin Invest 2009, 39:980-985

47. Chen M, Li Y, Yang T, Wang Y, Bai Y, Xie X: ADMA induces monocyte adhesion via activation of chemokine receptors in cultured THP-1 cells. Cytokine 2008, 43:149-159

48. Chen YH, Xu X, Sheng MJ, Zheng Z, Gu Q: Effects of asymmetric dimethylarginine on bovine retinal capillary endothelial cell proliferation, reactive oxygen species production, permeability, intercellular adhesion molecule-1, and occludin expression. Mol Vis 2011, 17:332-340

49. Takeuchi M, Makita Z, Yanagisawa K, Kameda K, Koike T: Detection of noncarboxymethyllysine and carboxymethyllysine advanced glycation end products (AGE) in serum of diabetic patients. Mol Med 1999, 5:393-405 